support from: Actelion, Roche, MSD, Consultant of: GlaxoSmithKline, VivaCell Biotechnology, Emerald Health Pharmaceuticals, Boehringer Ingelheim, Roche, Speakers bureau: Actelion, GlaxoSmithKline, Roche DOI: 10.1136/annrheumdis-2020-eular.6566

\section{AB0603 PDGFA AS A POTENTIAL BLOOD MARKER IN DSSC}

K. Romanowska-Prochnicka ${ }^{1,2}$, A. Wajda ${ }^{3}$, A. Paradowska-Gorycka ${ }^{3}$, A. FelisGiemza $^{2}$, E. Walczuk ${ }^{3}$, M. Olesińska ${ }^{2}$ on behalf of No. ${ }^{1}$ Medical University of Warsaw, Department of General and Experimental Pathology with Centre for Preclinical Research and Technology (CEPT), Warsaw, Poland; ${ }^{2}$ National Institute of Geriatrics, Rheumatology and Rehabilitation, Warsaw, Poland, Department of Systemic Connective Tissue Diseases, Warsaw, Poland; ${ }^{3}$ National Institute of Geriatrics, Rheumatology, and Rehabilitation, Department of Molecular Biology, Warsaw, Poland

Background: PDGF is a potential important factor in the pathogenesis of scleroderma. PDGF is almost undetectable in healthy skin or lung. Immunohistochemical studies have revealed increased presence of PDGF $\alpha$ and PDGF $\beta$ receptors in scleroderma skin biopsies.

Objectives: The aim of this study was to determine the mRNA level of IFNa1, $I L-4, T G F \beta 1, T G F \beta 2, P D G F a, P D G F \beta, T N F a$ in whole blood in SSc patients in the aspect of clinical

Methods: A group of 14 patients (50\% were women) with systemic sclerosis based on EULAR / ACR 2013 criteria was included in the study. The modified Rodnan Skin Score (mRSS) was evaluated by same assistant at the beginning of the study and six months later. DLCO, HRCT, echocardiography and NFC were measured. Gene expression was determined using validated TaqMan probes in qPCR. Constitutive mRNA level of selected genes was analyzed using $\Delta \mathrm{Ct}$ method. Comparison between different groups of patients was determined using non-parametric Mann-Whitney $U$ test. Correlation was analyzed using non-parametric Spearman test. Results: The mean age of the patients was $60 \pm 15.66 .100 \%$ of patients had organ involvement as pulmonary fibrosis. $78 \%$ - had active changes -features of ground glass.

$64 \%$ of patients had mild mRSS-1-10 skin involvement, $36 \%$ had moderate to severe skin involvement. In SSc patients TGF 1 and IFNa1 revealed the highest level of expression in comparison to other analyzed genes. Additionally, very high and significant correlation between TNF $a$ and TGF $\beta 1(r=0.7 \mathrm{p}=0.004)$ has been noted. High and significant correlation between mRNA PDGF $\beta$ and TNFa levels have been observed. We did not reveal significant differences in analyzed genes expression when compare limited and diffuse SSc. Nevertheless, patients with dSSc were characterized by higher level of IFNa1 (almost 2 times) and TGF $\beta 1$. On the border of significance higher PDGFa mRNA level was observed in dSSc patients when compared to ISSc. Average PDGFa expression is higher in SSc patients with Scl70 positive than than in patients without Scl70 $(p=0.04)$.

In the aspect of clinical parameters, patients with ESR $\leq 12 \mathrm{~mm} / \mathrm{h}$ revealed almost 6 times higher level of IFNa1 $(\mathrm{p}=0.01)$ in comparison to the patient with $E S R>12 \mathrm{~mm} / \mathrm{h}$. Patients with mRSS above10 points revealed significantly higher of PDGFa expression in comparison to patients with $\mathrm{mRSS} \leq 10(p=0.04)$. In these group of patients CRP and ESR were not different significantly.

In the case of patients with active fibrosis (ground glass) in HRCT IFNa1 expression was almost 2.5-times higher than in patients with HRCT non-active. Significantly higher PDGFa has been revealed in patients with active HRCT when compared with patient with non-active HRCT. Nevertheless these two groups did not differ in ESR or OB parameter.

SSc patients in active phase of NFC revealed almost 3 times higher level of IFNa1 expression in comparison with the patient in late phase.

Conclusion: The mRNA level of PDGFa may be a potential blood marker to predict worse prognosis in scleroderma

References:

[1] Rheumatology (Oxford). 2008 Oct;47 Suppl 5:v2-4. doi: 10.1093/ rheumatology/ken265.

Role of PDGF in fibrotic diseases and systemic sclerosis.

Trojanowska M1.

Acknowledgments: No

Disclosure of Interests: None declared

DOI: 10.1136/annrheumdis-2020-eular.5590

\section{$\mathrm{AB} 0604$ \\ EVALUATION OF PATIENTS WITH ANTISYNTHETASE SYNDROME AND INTERSTITIAL LUNG DISEASE BASED ON THE RADIOLOGICAL PATTERN}

A. Ruiz Roman ${ }^{1}$, J. A. Rodriguez Portal' . 'Hospital Virgen del Rocío, Rheumatology, Seville, Spain; ${ }^{2}$ Hospital Virgen del Rocío, Pneumology, Seville, Spain

Background: Antisynthetase syndrome is an autoimmune rheumatologic disease characterized by the presence of specific antibodies that are known as antisynthetase antibodies and with a varied clinic, including arthritis, myositis or interstitial lung disease (ILD), among others. ILD is the manifestation that associates the highest morbi / mortality and can occur with different characteristic radiological patterns: usual interstitial pneumonia (UIP), non-specific interstitial pneumonia (NSIP), organized pneumonia (OP)

Objectives: Describe the clinical, serological and prognostic characteristics of patients with antisynthetase syndrome with interstitial pulmonary involvement (ILD) based on their radiological pattern.

Methods: Retrospective descriptive study of patients with a clinical diagnosis of antisynthetase syndrome in which they show positive for some antisynthetase antibody and with clinical follow-up of at least 6 months in a reference center consultation between the period from January 2008 to September 2019. In those patients who presented interstitial lung involvement clinical, analytical and prognostic variables were evaluated (including spirometry) based on the radiological pattern presented by high-resolution computed tomography (HRCT) of the chest Results: 32 patients ( 24 women and 8 men) were included in the study. 7 cases $(21.9 \%)$ did not present pulmonary involvement, while the remaining 25 cases (78.1\%) presented with interstitial lung involvement. Of the patients diagnosed with ILD, 4 cases $(12.5 \%)$ had an interstitial pattern UIP, 17 cases $(53.1 \%)$ had an interstitial pattern of NSIP and another $4(12.5 \%)$ interstitial pattern of OP. From an analytical point of view, the most frequent antisynthetase antibody in our sample was antiJo1 with 29 cases (96.9\%) and less frequently the antiPL12 antibody with 2 cases and antiPL7 with 1 case. The specific characteristics depending on the type of EPID pattern are summarized in Table 1.

Table 1.

\begin{tabular}{|c|c|c|c|}
\hline \multirow[t]{2}{*}{ CHARACTERISTICS } & \multicolumn{3}{|c|}{ ILD PATTERN } \\
\hline & $\begin{array}{l}\text { Pattern UIP } \\
\quad \mathrm{N}=4\end{array}$ & $\begin{array}{c}\text { Pattern NSIP } \\
\quad \mathrm{N}=17\end{array}$ & $\begin{array}{l}\text { Pattern OP } \\
\quad \mathrm{N}=4\end{array}$ \\
\hline Gender (Female) n (\%) & $4(100)$ & $11(64.7)$ & $3(75.0)$ \\
\hline Age at diagnosis & $47.5 \pm 12.9$ & $52.9 \pm 10.5$ & $42.0 \pm 12.9$ \\
\hline Myositis, $n(\%)$ & $2(50.0)$ & $13(76.5)$ & $4(100)$ \\
\hline Arthritis, n (\%) & $3(75.0)$ & $15(88.2)$ & $3(75.0)$ \\
\hline Fever, $\mathrm{n}(\%)$ & $1(25.0)$ & $9(52.9)$ & $2(50.0)$ \\
\hline Mechanic's hands, n(\%) & $2(50.0)$ & $7(41.2)$ & $2(50.0)$ \\
\hline Raynaud's phenomen, n (\%) & $1(25.0)$ & $8(47.1)$ & $1(25.0)$ \\
\hline Skin involvement, $n(\%)$ & $2(50.0)$ & $10(58.8)$ & $4(100)$ \\
\hline \multicolumn{4}{|l|}{ Treatment, n(\%) } \\
\hline Glucocorticoids & $4(100)$ & $16(94.1)$ & $4(100)$ \\
\hline Immunosuppresants & $4(100)$ & $16(94.1)$ & $4(100)$ \\
\hline Biological therapy & $1(25.0)$ & $8(47.1)$ & $2(50.0)$ \\
\hline Drug's number (mean, SD) & $3 \pm 0.8$ & $5 \pm 2.9$ & $4.7 \pm 1.8$ \\
\hline \multicolumn{4}{|l|}{ Antisynthetase antibodies, $n(\%)$} \\
\hline Anti Jo1 & $3(75.0)$ & $15(88.2)$ & $4(100)$ \\
\hline PL7 & $0(0)$ & $1(5.9)$ & $0(0)$ \\
\hline PL12 & $1(25.0)$ & $1(5.9)$ & $0(0)$ \\
\hline Ro52 & $3(75.0)$ & $14(82.4)$ & $2(50.0)$ \\
\hline RF positive & $1(25.0)$ & $4(23.5)$ & $1(25.0)$ \\
\hline CK increase & $2(50.0)$ & $11(64.7)$ & $4(100)$ \\
\hline Aldolase increase & $2(50.0)$ & $12(70.6)$ & $4(100)$ \\
\hline \multicolumn{4}{|l|}{$\begin{array}{l}\text { Functional respiratory tests: } \\
\text { mean }(\mathrm{Cl} 95 \%)\end{array}$} \\
\hline Variation DLCOc(\%) & $-4.4(-12.5,3.7)$ & $-2.5(-6.1,1.0)$ & $7.4(-10.6,25.3)$ \\
\hline Variation $\mathrm{FVC}(\%)$ & $-1.1(-12.8,9.1)$ & $6.0(3.2,8.8)$ & $7.5(-9.5,24.6)$ \\
\hline Variation FEV1(\%) & $0(0-2.25)$ & $5.0(1.9,8.3)$ & $7.35(-18.0,32.7)$ \\
\hline Variation FEV1/FVC(\%) & $0.5(-7.1,8.2)$ & $-1.3(-2.22,-0.37)$ & $-0.4(-12.88,11.98)$ \\
\hline
\end{tabular}

Conclusion: ILD is a frequent and serious manifestation that can occur in patients with antisynthetase syndrome, which may have different radiological patterns. In our series, the most observed radiological pattern has been the NSIP pattern with $68 \%$. The pattern most associated with the Ro 52 antibody was the UIP pattern and the NSIP pattern. As for the group that required the largest numbers of drugs (including biological therapy) it was the NSIP pattern. When evaluating changes in respiratory function tests, the pattern that shows a tendency to improve over time is organized pneumonia with improvement of the DLCO, FVC, FEV1, FEV1 / FVC, while the UIP pattern showed a worsening of the DLCO and the FVC.

Disclosure of Interests: None declared

DOI: 10.1136/annrheumdis-2020-eular.3584

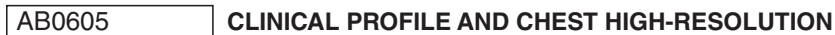 COMPUTED TOMOGRAPHY (HRCT) FINDINGS IN PATIENTS WITH CONNECTIVE TISSUE DISEASES AND INTERSTITIAL LUNG DISEASE: EXPERIENCE OF A SINGLE REFERENCE RHEUMATOLOGY CENTER}

$\underline{\text { I. Rusu }}^{1}$, L. Muntean ${ }^{1,2}$, M. M. Tamas ${ }^{1,2}$, I. Felea ${ }^{2}$, L. Damian ${ }^{1}$, I. Filipescu ${ }^{1,2}$, S. P. Simon ${ }^{1,2}$, C. Pamfil ${ }^{2}$, C. M. Marinescu ${ }^{3}$, M. A. Man ${ }^{2,4}$, C. Csutak ${ }^{2,3}$, S. Rednic ${ }^{1,2}$. $^{1}$ Emergency Clinical County Hospital, Rheumatology, ClujNapoca, Romania; "'luliu Hatieganu" University of Medicine and Pharmacy, Cluj-Napoca, Romania; ${ }^{3}$ Emergency Clinical County Hospital, Radiology, 
Cluj-Napoca, Romania; ${ }^{4}$ Pulmonology Hospital “Leon Daniello, Cluj-Napoca, Romania

Background: Interstitial lung disease (ILD) is a common manifestation of connective tissue diseases (CTDs), and is associated with significant morbidity and mortality. Chest high-resolution computed tomography (HRCT) play an important role in the diagnosis of ILD and may provide prognostic information.

Objectives: We aimed to characterize the clinical profile and chest HRCT abnormalities and patterns of patients diagnosed with CTDs and ILD.

Methods: In this retrospective, observational study we included 80 consecutive patients with CTDs and ILD referred to a tertiary rheumatology center between 2015 and 2019. From hospital charts we collected clinical data, immunologic profile, chest HRCT findings. HRCT patterns were defined according to new international recommendations.

Results: Out of 80 patients, $64(80 \%)$ were women, with a mean age of 55 years old. The most common CTD associated with ILD was systemic sclerosis (38.8\%), followed by polymyositis (22.5\%) and rheumatoid arthritis (18.8\%). The majority of patients had dyspnea on exertion $(71.3 \%)$, bibasilar inspiratory crackles were present in $56.3 \%$ patients and $10 \%$ had clubbing fingers. Antinuclear antibodies (ANA) were present in $78.8 \%$ patients, and the most frequently detected autoantibodies against extractable nuclear antigen were anti-Scl 70 (28.8\%), followed by anti-SSA (anti-Ro, 17.5\%), anti-Ro52 (11.3\%) and anti-Jo $(7.5 \%)$. Intravenous cyclophosphamide therapy for $6-12$ months was used in $35 \%$ of patients, while $5 \%$ of patients were treated with mycophenolate mofetil.

The most frequent HRCT abnormalities were reticular abnormalities and ground glass opacity. Non-specific interstitial pneumonia (NSIP) was identified in $46.3 \%$ CTDs patients. A pattern suggestive of usual interstitial pneumonia (UIP) was present in $32.5 \%$ patients, mainly in patients with systemic sclerosis. In $21.3 \%$ patients the HRCT showed reticulo-nodular pattern, micronodules and other abnormalities, not diagnostic for UIP or NSIP pattern.

Conclusion: Nonspecific interstitial pneumonia (NSIP) is the most common HRCT pattern associated with CTDs. Further prospective longitudinal studies are needed in order to determine the clinical and prognostic significance of various HRCT patterns encountered in CTD-associated ILD and for better patient management.

References:

[1] Ohno Y, Koyama H, Yoshikaua T, Seki S. State-of-the-Art Imaging of the Lung for Connective Tissue Disease (CTD). Curr Rheumatol Rep. 2015;17(12):69.

[2] Walsh SLF, Devaraj A, Enghelmeyer JI, Kishi K, Silva RS, Patel N, et al. Role of imaging in progressive-fibrosing interstitial lung diseases. Eur Respir Rev. 2018;27(150)

Disclosure of Interests: None declared

DOI: 10.1136/annrheumdis-2020-eular.3758

\section{AB0606 SYSTEMIC SCLEROSIS - ARE PATIENTS WITH CALCINOSIS DIFFERENT FROM THOSE WHO DO NOT HAVE IT?}

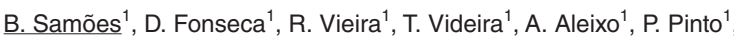
M. Guerra ${ }^{1} .{ }^{1}$ Centro Hospitalar Vila Nova de Gaia, Rheumatology, Vila Nova de Gaia, Portugal

Background: Systemic Sclerosis (SS) is a heterogenous disease with a broad range of organ involvement. Calcinosis is a common problem and although it may affect almost any body tissue, it is typically seen in the limbs. ${ }^{1}$ Its presence relates with higher risk of digital ulcers and infection. ${ }^{2}$ It is still unknown whether patients with calcinosis also have other clinical features that differentiate them from the remaining.

Objectives: To determine the prevalence of calcinosis in a SS cohort and to evaluate if its presence relates with specific clinical features.

Methods: A cross-sectional study was conducted evaluating a cohort of SS patients. Plain radiographs were taken to assess calcinosis at elbows, hands, knees and feet. Clinical data was obtained and analyzed using IBM SPSS Statistics $26 \circledast$.

Results: We included 25 patients, 21 females [ $n=21(84 \%)$ ], median (min, $\max )$ age was $58(27,75)$ years-old. Regarding disease classification, 16 $(64 \%)$ had limited SS, 4 (16\%) had diffuse SS, 3 (12\%) had overlap syndrome and $2(8 \%)$ had early SS. Ten $(40 \%)$ patients had radiological calcinosis in at least one site, seven of which $(70 \%)$ were subclinical. The most affected areas were knees and hands $[n=6(24 \%)]$. Table 1 summarizes the clinical characteristics of patients with and without calcinosis. Limited SS was significantly more prevalent in the calcinosis group $[n=9(90 \%)$ vs. $n=7(46.7 \%), p=0.04]$. All patients had Raynaud phenomenon [ $n=10(100 \%)$ vs. $15(100 \%)]$. Current or past digital ulcers $[n=5(50 \%)$ vs. $n=6(40 \%), p=0.697]$, telangiectasias $[n=9$ $(90 \%)$ vs. $n=11(73.3 \%), p=0.615]$, pulmonary hypertension $[n=2(20 \%)$ vs. $n=1(6.7 \%), p=0.550]$ and esophageal involvement $n=6(60 \%)$ vs. $n=6(40 \%)$, $p=0.428]$ were more frequent in the calcinosis group but with no statistical significance. Although late capillaroscopic pattern was more frequent in the calcinosis group, there was no statistical significance difference $[n=4(40 \%)$ vs. $n=1(6.7 \%), p=0.121]$. Seropositivity for centromere-B antibodies was more frequent in the calcinosis group but with no statistical significance $[n=7(70 \%)$ vs. $n=8(53.3 \%), p=0.678]$.

Table 1. Demographic and clinical data of patients with and without calcinosis.

\begin{tabular}{lccc}
\hline Demographic and clinical data & Calcinosis (n=10) & No calcinosis (n=15) & p-value \\
\hline Female gender, $\mathrm{n}(\%)$ & $9(90)$ & $12(80)$ & 0.626 \\
Age (years), median [min,max] & $68.5[27,75]$ & $52[36,73]$ & 0.129 \\
Cutaneous classification & & & \\
Limited, $\mathrm{n}(\%)$ & $9(90)$ & $7(46.7)$ & 0.04 \\
Diffuse, $\mathrm{n}(\%)$ & $1(10)$ & $3(20)$ & 0.626 \\
Early, $\mathrm{n}(\%)$ & $0(0)$ & $2(13.3)$ & 0.500 \\
Overlap, $\mathrm{n}(\%)$ & $0(0)$ & $3(20)$ & 0.250 \\
Clinical manifestations & & & \\
Current or previous digital ulcers, $\mathrm{n}(\%)$ & $5(50)$ & $6(40)$ & 0.697 \\
Interstitial lung disease, $\mathrm{n}(\%)$ & $2(20)$ & $4(26.7)$ & 1.000 \\
Pulmonary hypertension, $\mathrm{n}(\%)$ & $2(20)$ & $1(6.7)$ & 0.550 \\
Arthritis, $\mathrm{n}(\%)$ & $2(20)$ & $3(20)$ & 1.000 \\
Calcinosis, $\mathrm{n}(\%)$ & $3(30)$ & $0(0)$ & 0.052 \\
Esophageal involvement, $\mathrm{n}(\%)$ & $6(60)$ & $6(40)$ & 0.428 \\
NFC patterns & & & \\
Non specific abnormalities, $\mathrm{n}(\%)$ & $1(10)$ & $3(20)$ & 0.626 \\
Early scleroderma, $\mathrm{n}(\%)$ & $1(10)$ & $1(6.7)$ & 1.000 \\
Active scleroderma, $\mathrm{n}(\%)$ & $3(30)$ & $10(58.8)$ & 0.111 \\
Late scleroderma, $\mathrm{n}(\%)$ & $1(6.7)$ & 0.121 \\
Autoantibodies & $4(40)$ & & \\
Centromere B, $\mathrm{n}(\%)$ & $7(70)$ & $8(53.3)$ & 0.678 \\
Scl-70, $\mathrm{n}(\%)$ & $1(10)$ & $4(26.7)$ & 0.615 \\
\hline
\end{tabular}

Conclusion: The prevalence of calcinosis was similar to that reported in literature $(18-49 \%)$. This study confirmed the association, already found in previous studies, between calcinosis and the limited form of SS and raises attention for the importance of calcinosis radiographic screening since there was a high prev alence of subclinical calcinosis. ${ }^{1}$ Although there were some clinical differences between patients with and without calcinosis, given the small cohort, statistical significance was not obtained. Larger studies are needed to increase statistica power.

References:

[1] Valenzuela A et al. Calcinosis in scleroderma. Curr Opin Rheumatol. 2018 Nov;30(6):554-561.

[2] Bartoli $F$ et al. Calcinosis in systemic sclerosis: subsets, distribution and complications. Rheumatology (Oxford). 2016 Sep;55(9):1610-4

Disclosure of Interests: None declared

DOI: 10.1136/annrheumdis-2020-eular. 1526

\begin{tabular}{|l} 
AB0607 \\
MYOSITIS-RELATED INTERSTITIAL LUNG \\
DISEASES: CLINICAL FEATURES, BIOMARKERS \\
AND AUTOANTIBODIES IN LATINOAMERICAN \\
PATIENTS
\end{tabular}

S. M. Sánchez Romo ${ }^{1}$, G. G. Sánchez Mendieta ${ }^{1}$, M. A. Villarreal-Alarcón ${ }^{1}$, I. D. J. Hernandez-Galarza ${ }^{1}$, D. Á. Galarza-Delgado ${ }^{1}{ }^{1}$ Hospital Universitario Dr. José Eleuterio González UANL, Rheumatology, Monterrey, Mexico

Background: The lung is one of the most common extra-muscular targets in idiopathic inflammatory myopathies (IIM) and interstitial lung disease (ILD) is a prevalent and often devastating manifestation of IIM ${ }^{1}$.

Objectives: To know the frequency of autoantibodies associated with IMM-ILD biomarkers, and their relation with clinical features in patients with IIM.

Methods: Adults with IIM were enrolled in a retrospective way. Demographics, clinical and laboratory features were registered. The determination of antibodies was perfomed by the Inmunoblot technique with Euroinmmun kit. Patients without a myositis antibody panel were excluded. The diagnosis of ILD was based on HRCT. Patients with anti-MDA5 antibodies and with ant Ro-52 antibodies associated with anti-ARS were considered as high risk group, those with anti-ARS, anti-U1-RNP, anti-PM/ Scl and anti-Ku antibodies as moderate risk and those with anti-Mi2, anti SRP and anti TIF1 antibodies as low risk ${ }^{2}$.

Results: Demographics characteristics are shown in table 1. We included 36 patients. Dermatomyositis (DM) was described in $69.4 \%$, polymyositis (PM) in $16.7 \%$ and antysnthetase syndrome (AAS) in $13.9 \%$. Out of the total of our patients, $30.6 \%$ had interstitial lung disease. The most frequent autoantibody was Anti Ro52 in 13 (36.1\%) patients and 44.4\% were in the high risk group. We analized our patients by the presence or absence of ILD and we found that 